\title{
TRANSLATED FUNCTIONS AND STATISTICAL INDEPENDENCE ${ }^{1}$
}

\author{
R. P. AGNEW AND M. KAC
}

1. Introduction. Professor Steinhaus ${ }^{2}$ proposed the question wheththere exists a function $f(t)$ defined in $(-\infty, \infty)$ such that, for each sequence $\lambda_{1}, \lambda_{2}, \cdots$ of different real numbers, the "translated" functions $f\left(t+\lambda_{1}\right), f\left(t+\lambda_{2}\right), \cdots$ form a sequence of statistically independent functions. ${ }^{3}$ We shall answer this question in the affirmative by giving concrete examples, and shall discuss some related problems.

2. Notation and lemmas. Let

$$
\mathcal{X}\{f(t)\}=\lim _{T \rightarrow \infty} \frac{1}{2 T} \int_{-T}^{T} f(t) d t
$$

denote as usual the mean value of $f(t)$ in case the limit exists. If $E$ is a point set on the line $(-\infty, \infty)$ and $g(t)$ is the characteristic function of $E$, the mean value $\mathcal{X}\{g(t)\}$ (if it exists) is called the relative measure of $E$ and will be denoted by $|E|$. A non-decreasing function $\sigma(\alpha)$ such that $\sigma(-\infty)=0, \sigma(+\infty)=1$ is called an asymptotic distribution function of $f(t)$ if

$$
|\underset{t}{E}\{f(t)<\alpha\}|=\sigma(\alpha)
$$

at each point of continuity of $\sigma(\alpha)$. A set $f_{1}(t), \cdots, f_{n}(t)$ of functions having asymptotic distribution functions $\sigma_{1}(\alpha), \cdots, \sigma_{n}(\alpha)$ is called statistically independent if

$$
\left|\underset{t}{E}\left\{f_{1}(t)<\alpha_{1} ; \cdots ; f_{n}(t)<\alpha_{n}\right\}\right|=\sigma_{1}\left(\alpha_{1}\right) \cdots \sigma_{n}\left(\alpha_{n}\right)
$$

for each set $\alpha_{1}, \cdots, \alpha_{n}$ of real numbers such that $\alpha_{k}$ is a point of continuity of $\sigma_{k}(\alpha)$. An infinite set of functions is called statistically independent if each finite subset is statistically independent.

Our proofs will be based on the following theorem of Kac and Steinhaus, loc. cit.

\footnotetext{
1 Presented to the Society, February 24, 1940.

${ }^{2}$ In a letter to one of the authors. The problem arose in connection with the theory of turbulence, but because of the outbreak of the war we have not been able to get details.

${ }^{3}$ See M. Kac and H. Steinhaus, Sur les fonctions indépendantes IV, Studia Mathematica, vol. 7 (1937), pp. 1-15; and P. Hartman, E. R. van Kampen, and A. Wintner, Asymptotic distributions and statistical independence, American Journal of Mathematics, vol. 61 (1939), pp. 477-486.
} 
Lemma 1. A set $f_{1}(t), \cdots, f_{n}(t)$ of bounded functions having absolutely continuous distribution functions is statistically independent if and only if the equality

$$
\mathcal{X}\left\{\prod_{j=1}^{n} f_{j}^{k_{j}}(t)\right\}=\prod_{j=1}^{n} \mathcal{X}\left\{f_{j}^{k_{j}}(t)\right\}
$$

holds for each set $k_{1}, k_{2}, \cdots, k_{n}$ of positive integer exponents.

Repeated use will be made of the following simple lemma.

LEMMA 2. If $\phi(t)$ is a real differentiable function and constants $\tau>0$ and $\alpha>0$ exist such that $\phi^{\prime}(t)$ is monotone and $\left|\phi^{\prime}(t)\right|>\alpha$ over each of the intervals $(-\infty,-\tau)$ and $(\tau, \infty)$, then

$$
\mathcal{X C}\left\{e^{i \phi(t)}\right\}=0 .
$$

Let $I_{1}, I_{2}$, and $I_{3}$ denote the integrals of $\exp i \phi(t)$ over $(-T,-\tau)$, $(-\tau, \tau)$, and $(\tau, T)$ respectively. Applying the second mean value theorem we obtain, when $T>\tau$,

$$
\begin{aligned}
I_{3}= & \int_{\tau}^{T} \frac{\phi^{\prime}(t) \cos \phi(t)}{\phi^{\prime}(t)} d t+i \int_{\tau}^{T} \frac{\phi^{\prime}(t) \sin \phi(t)}{\phi^{\prime}(t)} d t \\
= & \frac{1}{\phi^{\prime}(\tau)} \int_{\tau}^{\xi_{1}} \phi^{\prime}(t) \cos \phi(t) d t+\frac{1}{\phi^{\prime}(T)} \int_{\xi_{1}}^{T} \phi^{\prime}(t) \cos \phi(t) d t \\
& +\frac{i}{\phi^{\prime}(\tau)} \int_{\tau}^{\xi_{2}} \phi^{\prime}(t) \sin \phi(t) d t+\frac{i}{\phi^{\prime}(T)} \int_{\xi_{2}}^{T} \phi^{\prime}(t) \sin \phi(t) d t,
\end{aligned}
$$

and evaluating the last four integrals gives $\left|I_{3}\right|<8 / \alpha$. Likewise $\left|I_{1}\right|<8 / \alpha$, and since $\left|I_{2}\right| \leqq 2 \tau$ our lemma follows.

If $\phi(t)$ is a real polynomial in $t$ of positive degree, Lemma 2 is obviously applicable. The same is true if $\phi(t)=\sum_{j=1}^{n} a_{\jmath} \exp \left(t+\lambda_{j}\right)^{2}$ where $\lambda_{1}, \cdots, \lambda_{n}$ are different real numbers and $a_{1}, \cdots, a_{n}$ are real coefficients not all 0 .

3. An example. We can now establish our main result.

THEOREM 1. The translations of the function

$$
f(t)=\cos e^{t^{2}}
$$

form a statistically independent set; that is, for each set $\lambda_{1}, \cdots, \lambda_{n}$ of different real numbers, the functions $f\left(t+\lambda_{1}\right), \cdots, f\left(t+\lambda_{n}\right)$ are statistically independent.

Our first step is to verify that (4) holds. We find that 


$$
\begin{aligned}
\prod_{j=1}^{n} \cos ^{k_{j}} e^{\left(t+\lambda_{j}\right)^{2}}= & \prod_{j=1}^{n} 2^{-k_{j}}\left[\exp \left(i e^{\left(t+\lambda_{j}\right)^{2}}\right)+\exp \left(-i e^{\left(t+\lambda_{j}\right)^{2}}\right)\right]^{k_{j}} \\
= & \prod_{j=1}^{n} 2^{-k_{j}} \sum_{\alpha_{j}=0}^{k_{j}} C_{k_{j}, \alpha_{j}}\left[\exp \left(i \alpha_{j} e^{\left(t+\lambda_{j}\right)^{2}}\right)\right] \\
& \cdot\left[\exp \left(-i\left(k_{j}-\alpha_{j}\right) e^{\left(t+\lambda_{j}\right)^{2}}\right)\right] \\
= & \prod_{j=1}^{n} 2^{-k_{j}} \sum_{\alpha_{j}=0}^{k_{j}} C_{k_{j}, \alpha_{j}} \exp i\left(2 \alpha_{j}-k_{j}\right) e^{\left(t+\lambda_{j}\right)^{2}} \\
= & 2^{-k_{1}} \cdots 2^{-k_{n}} \sum_{\alpha_{1}=0}^{k_{1}} \cdots \sum_{\alpha_{n}=0}^{k_{n}} C_{k_{1}, \alpha_{1}} \cdots C_{k_{n}, \alpha_{n}} \\
& \cdot \exp i \sum_{j=1}^{n}\left(2 \alpha_{j}-k_{j}\right) e^{\left(t+\lambda_{j}\right)^{2}} .
\end{aligned}
$$

Since $\mathcal{X}\{f\}$ is an additive homogeneous operation, we can apply Lemma 2 to show that

$$
\mathscr{X}\left\{\prod_{j=1}^{n} \cos ^{k_{j}} e^{\left(t+\lambda_{j}\right)^{2}}\right\}=0
$$

if at least one of $k_{1}, k_{2}, \cdots, k_{n}$ is odd; but

$$
\mathcal{X}\left\{\prod_{j=1}^{n} \cos ^{k_{j}} e^{\left(t+\lambda_{j}\right)^{2}}\right\}=\prod_{j=1}^{n} 2^{-k_{j} C_{k_{j}, k_{j} / 2}}
$$

if $k_{1}, k_{2}, \cdots, k_{n}$ are all even. Noticing that the particular case $n=1$ gives

$$
\begin{aligned}
\mathscr{X}\left\{\cos ^{k} e^{(t+\lambda)^{2}}\right\} & =0, & k \text { odd, } \\
& =2^{-k} C_{k, k / 2}, & k \text { even, }
\end{aligned}
$$

we see that the mean value of the product is the product of the mean values as in (4). Moreover the fact that the moments of $f(t) \equiv \cos \exp (t+\lambda)^{2}$ are the same as the moments of $\cos t$ implies $^{4}$ that the distribution function of $f(t)$ is the same as that of $\cos t$ and hence is absolutely continuous. Therefore we can apply Lemma 1 to obtain the conclusion of Theorem 1.

4. Functions which oscillate less rapidly. The function $\cos \exp t^{2}$ of Theorem 1 oscillates very rapidly as $|t| \rightarrow \infty$. A natural question

${ }^{4}$ A. Wintner, Über die statistiche Unabhängigkeit der asymptotischen Verteilungsfunktionen inkommensurabler Partialschwingungen, Mathematische Zeitschrift, vol. 36 (1933), pp. 618-629. 
arises whether $\exp t^{2}$ can be replaced by a positive integer power of $t$, say $t^{m}$. If we put $f(t)=\cos t^{m}$, then the answer to the question of statistical independence of the translations $f\left(t+\lambda_{1}\right), f\left(t+\lambda_{2}\right), \cdots$ is only partially positive.

Theorem 2. If $f(t)=\cos t^{m}, m$ being a positive integer, then each set of $m$ different translations $f\left(t+\lambda_{1}\right), \cdots, f\left(t+\lambda_{m}\right)$ is a statistically independent set; but $(m+1)$ different numbers $\lambda_{1}, \cdots, \lambda_{m+1}$ can be chosen in such a way that the corresponding set of $(m+1)$ translations is not statistically independent.

Proof of this theorem is similar to that of Theorem 1. For each $n=1,2, \ldots$ we find on setting

$$
P_{n}(t)=\prod_{j=1}^{n} \cos ^{k_{j}}\left(t+\lambda_{j}\right)^{m}
$$

that

$$
\mathcal{M C}\left\{P_{n}(t)\right\}=2^{-k_{1}} \cdots 2^{-k_{n}} \sum_{\alpha_{1}=0}^{k_{1}} \cdots \sum_{\alpha_{n}=0}^{k_{n}} C_{k_{1}, \alpha_{1}} \cdots C_{k_{n}, \alpha_{1}} \mathcal{J C}\left\{e^{i \phi(t)}\right\}
$$

where $\phi(t) \equiv \phi\left(\lambda_{1}, \cdots, \lambda_{n} ; k_{1}, \cdots, k_{n} ; \alpha_{1}, \cdots, \alpha_{n} ; t\right)$ is given by

$$
\phi(t)=\sum_{j=1}^{n}\left(2 \alpha_{j}-k_{j}\right)\left(t+\lambda_{j}\right)^{m}=\sum_{s=0}^{m}\left[C_{m, s} \sum_{j=1}^{n}\left(2 \alpha_{j}-k_{j}\right) \lambda_{j}^{s}\right] t^{m-s}
$$

Applying Lemma 2, we see that $\mathcal{X}\{\exp i \phi(t)\} \neq 0$ if and only if

$$
\sum_{j=1}^{n}\left(2 \alpha_{j}-k_{j}\right) \lambda_{j}^{s}=0, \quad s=0,1, \cdots, m-1 .
$$

In case $n=m,(13)$ is a system of $m$ homogeneous linear equations in $m$ quantities $\left(2 \alpha_{j}-k_{j}\right)$ whose determinant (Vandermonde's determinant) does not vanish; and if (13) holds, then $2 \alpha_{j}=k_{j}$ when $j=1,2, \cdots, m$. Hence, in case $n=m, \mathscr{X}\{\exp i \phi(t)\}=0$ unless $k_{1}, \cdots, k_{m}$ are even and $\alpha_{j}=k_{j} / 2$ when $j=1,2, \cdots, m$. Therefore, in case $n=m$, formulas analogous to (7) and (8) hold and statistical independence of $f\left(t+\lambda_{1}\right), \cdots, f\left(t+\lambda_{m}\right)$ follows as in the proof of Theorem 1.

In case $m>1$ and $\lambda_{1}, \lambda_{2}, \cdots, \lambda_{n}$ are linearly independent (in the sense that no one of $\lambda_{1}, \cdots, \lambda_{n}$ is a linear combination with rational coefficients of the remaining) then (13) again implies that $2 \alpha_{j}=k_{j}$ and we conclude that $f\left(t+\lambda_{1}\right), \cdots, f\left(t+\lambda_{n}\right)$ are statistically independent. In case $m>1$, this shows existence of infinite sets of statistically independent translations of $\cos t^{m}$. 
We complete the proof of Theorem 2 by proving the following theorem.

THEOREM 3. If $m$ is a positive integer and $\lambda_{1}, \lambda_{2}, \cdots, \lambda_{m+1}$ are $m+1$ different rational numbers, then the set of $(m+1)$ translations

$$
\cos \left(t+\lambda_{1}\right)^{m}, \cos \left(t+\lambda_{2}\right)^{m}, \cdots, \cos \left(t+\lambda_{m+1}\right)^{m}
$$

is not statistically independent.

We use the formulas obtained by setting $n=m+1$ in (10), (11), (12), and (13). The system

$$
\sum_{j=1}^{m+1} \lambda_{j}^{s} x_{j}=0, \quad s=0,1, \cdots, m-1,
$$

of $m$ equations in $(m+1)$ unknowns $x_{1}, \cdots, x_{m+1}$ is such that if a nonzero rational value is assigned to one of the $x$ 's, then the remaining $x$ 's are uniquely determined and are rational. It follows that (15) has non-trivial solutions in integers. Let $X$ denote the set of points $x \equiv\left(x_{1}, \cdots, x_{m+1}\right)$ in $(m+1)$-dimensional space for which $x_{1}, \ldots, x_{m+1}$ are integers, not all 0 , satisfying (15). For each $x \in X$, let $\|x\|^{2}=x_{1}^{2}+\cdots+x_{m+1}^{2}$. Since $\|x\|^{2}$ is necessarily a positive integer, the minimum value of $\|x\|^{2}$ for $x \in X$ is an integer $R>0$. Choose $y \equiv\left(y_{1}, \cdots, y_{m+1}\right) \in X$ such that $\|y\|^{2}=R$, and let $k_{1}, \cdots, k_{m+1}$ be defined by

$$
k_{j}=\left|y_{j}\right|, \quad j=1,2, \cdots, m+1 .
$$

If $0 \leqq \alpha_{j} \leqq k_{j}$ for each $j=1,2, \cdots, m+1$ while $0<\alpha_{j}<k_{j}$ for at least one $j$, then

$$
\sum_{j=1}^{m+1}\left(2 \alpha_{j}-k_{j}\right)^{2}<\sum_{j=1}^{m+1} k_{j}^{2}=\sum_{j=1}^{m+1} y_{j}^{2}=\|y\|^{2}=R
$$

and the minimal property of $R$ implies that the point

$$
\left(2 \alpha_{1}-k_{1}, 2 \alpha_{2}-k_{2}, \cdots, 2 \alpha_{n+1}-k_{n+1}\right)
$$

is not in the set $X$; this means that at least one of the equations in (13) fails to hold, and accordingly $\mathcal{X}\{\exp i \phi(t)\}=0$. The remaining $2^{m+1}$ terms in the sum (11) are those for which each $\alpha_{j}$ is either 0 or $k_{j}$ and accordingly (18) is a point on the sphere (hypersphere) $S$ with center at $(0, \cdots, 0)$ and radius $R^{1 / 2}$. But the set $X$, being on each of $m$ independent planes (hyperplanes), is on their line of intersection; hence at most two of the points on $S$ can be in the set $X$. It follows that the point (18) fails to be in $X$, and hence that $\mathcal{X}\{\exp i \phi(t)\}=0$, 
unless the indices $\alpha_{1}, \cdots, \alpha_{n+1}$ are such that (i) $2 \alpha_{j}-k_{j}=y_{j}$ for each $j=1, \cdots, m+1$ or (ii) $2 \alpha_{j}-k_{j}=-y_{j}$ for each $j=1, \cdots, m+1$. In case (i), (13) holds so that $\phi(t)$ is independent of $t$, and we see that $\phi(t)=\theta$ where

$$
\theta=\sum_{j=1}^{m+1} y_{j} \lambda_{j}^{m}
$$

in case (ii), $\phi(t)=-\theta$. Therefore

$$
\begin{aligned}
\mathscr{X}\left\{\prod_{j=1}^{m+1} \cos ^{k_{i}}\left(t+\lambda_{j}\right)^{m}\right\} & =2^{-k_{1}} \cdots 2^{-k_{m+1}}\left(\mathcal{X}\left\{e^{i \theta}\right\}+\mathcal{X}\left\{e^{-i \theta}\right\}\right) \\
& =2^{-k_{1}} \cdots 2^{-k_{m+1}}(2 \cos \theta) \neq 0,
\end{aligned}
$$

the last step being a consequence of the fact that the right member of (19) is rational and hence that $\theta$ cannot be an odd multiple of $\pi / 2$.

At least one of $k_{1}, k_{2}, \cdots, k_{n+1}$ must be odd, for otherwise $z \equiv\left(y_{1} / 2, \cdots, y_{m+1} / 2\right)$ would be a point of $X$ with $\|z\|^{2}=\|y\|^{2} / 4$ $=R / 4<R$; therefore

$$
\prod_{j=1}^{m+1} \mathcal{X}\left\{\cos ^{k_{j}}\left(t+\lambda_{j}\right)^{m}\right\}=0 .
$$

Comparing (20) and (21), we see that (4) fails when $n=m+1$ and $f_{j}(t)=\cos \left(t+\lambda_{j}\right)^{m}$; hence Lemma 1 implies that the $(m+1)$ translations in (14) are not statistically independent. This completes proof of Theorems 3 and 2 .

Anyone who wishes to check Theorem 3 by consideration of a simple special case will find that if $m=2, \lambda_{1}=1, \lambda_{2}=2, \lambda_{3}=3$ then $y \equiv\left(y_{1}, y_{2}, y_{3}\right)$ may be either $(1,-2,1)$ or $(-1,2,-1)$; that $k_{1}=1$, $k_{2}=2, k_{3}=1$; and that elementary formulas for products of cosines and use of Lemma 2 gives

$$
\mathcal{X C}\left\{\cos (t+1)^{2} \cos ^{2}(t+2)^{2} \cos (t+3)^{2}\right\}=8^{-1} \cos 2,
$$

which is a special case of (20).

5. Other functions. Our methods can be used to show that certain rapidly oscillating functions $f(t)$ other than the function $\cos \exp t^{2}$ of $\$ 3$ have statistically independent translations. Our first solution of the problem of $\$ 1$ was a "direct" (but too tedious to be given here) proof of the fact that the set of all translations of the function $\cos \exp \exp t^{2}$ is statistically independent. Our "direct" method uses the definition of statistical independence rather than the criterion of Lemma 1, and applies only to functions which oscillate very rapidly. 
To handle such functions as $\cos t^{m}$, where the question of statistical independence of translations is more delicate, we need the criterion of Lemma 1.

6. Applications. As soon as statistical independence of a set of functions is established, we can apply the general theory of addition of independent random variables. ${ }^{5}$ As an illustration, we mention the following result: If $f(t)=\cos \exp t^{2}$, and $\lambda_{1}, \lambda_{2}, \cdots$ is a sequence of different real numbers, then

(23) $\lim _{n \rightarrow \infty}\left|\underset{t}{E}\left\{f\left(t+\lambda_{1}\right)+\cdots+f\left(t+\lambda_{n}\right)<\alpha n^{1 / 2}\right\}\right|=\frac{1}{\pi^{1 / 2}} \int_{-\infty}^{\alpha} e^{-u^{2}} d u$.

CORNell University

${ }^{5}$ See Kac and Steinhaus, loc. cit., and further references given there. Also P. Lévy, Thêorie de l'Addition des Variables Aléatoires, Paris, Gauthier-Villars, 1937. 